\title{
Analysis of Psychological Symptoms in a Retrospective Cohort of Children and Adolescents with Neuropathic and Non-Neuropathic Chronic Pain
}

\author{
David J Krodel ${ }^{1 *}$, Anshu S Hemrajani ${ }^{1}$, John V Lavigne ${ }^{2}$, Ravi D Shah ${ }^{1}$ and Bonnie S Essner ${ }^{2}$ \\ ${ }^{1}$ Department of Anesthesiology, Northwestern University Feinberg School of Medicine, USA \\ ${ }^{2}$ Department of Psychiatry and Behavioral Sciences, Northwestern University Feinberg School of Medicine, USA
}

Submission: February 12, 2018; Published: February 23, 2018

*Corresponding author: David J Krodel, Department of Anesthesiology, Northwestern University Feinberg School of Medicine, Department of Pediatric Anesthesiology, Division of Pain Medicine, Ann \& Robert H. Lurie Children's Hospital of Chicago, 225 E Chicago Ave, Box 19, Chicago, IL, USA 60611, Email: dkrodel@luriechildrens.org

\section{Abstract}

Background: We aimed to determine what psychiatric symptoms, if any, were present in a pediatric neuropathic pain sample, and if they differed from the symptoms observed in a non-neuropathic pain sample and from norms.

Methods: Records from a retrospective cohort of 104 children at a multidisciplinary, single center, pediatric pain clinic were examined. Patients were categorized by diagnosis (neuropathic and non-neuropathic comparison group). Child \& Adolescent Symptom Inventory scores, Numeric Rating Scale pain scores, duration of treatment, and demographic data were collected. We hypothesized that patients with neuropathic pain would differ from the comparison group in respect to psychiatric symptoms.

Results: Demographically the neuropathic and comparison groups were similar in number of females (neuropathic $71 \%$, comparison $75 \%$, $\mathrm{P}$ $=.67$ ), and Medicaid beneficiaries (neuropathic $25 \%$, comparison $30 \%, \mathrm{P}=.66$ ). Differences were noted in symptoms of generalized anxiety (neuropathic 61.9, $\mathrm{SD} \pm 10.0$, comparison $55.8, \mathrm{SD} \pm 8.6, \mathrm{P}<0.001$ ) and attention deficit hyperactivity disorder (neuropathic 52.3, SD \pm 5.3 , comparison 55.3, $\mathrm{SD} \pm 8.9, \mathrm{P}=.05$ ). Neuropathic pain patients reported lower post-treatment pain scores than comparisons (neuropathic 4.0 $\mathrm{SD} \pm 2.7$, comparison $5.3, \mathrm{SD} \pm 3.0, \mathrm{P}=0.04$ ). Higher final pain scores are significantly correlated with higher initial pain and higher generalized anxiety.

Conclusions: Measures of anxiety were lower in children with neuropathic pain than the non-neuropathic comparison group.

\section{Introduction}

The co-occurrence of psychological distress and pediatric chronic pain is well established [1-5], but estimates of the prevalence of psychiatric disorders among youth with chronic pain conditions vary considerably (20-75\%) [6-8]. The association of psychiatric symptoms and pediatric neuropathic pain conditions including complex regional pain syndrome (CRPS) has been even more difficult to characterize. Case reports, anecdotal evidence, and small cohort studies have supported the notion that the physical manifestations of CRPS may be primarily driven by psychological trauma or distress [9-11]. Another study found that children with CRPS in fact had normal levels of depression and anxiety and no overall group differences on these measures among children with CRPS, headache, back pain, or abdominal pain [12]. Also unclear, and controversial, is whether psychiatric symptoms are more heavily involved in the onset and continuation of different chronic pain syndromes (e.g. CRPS, musculoskeletal pain, abdominal pain, and headache). This study of psychological symptoms in adolescents with neuropathic and non-neuropathic chronic pain contributes to this relatively sparse literature.

Regardless of the role of psychiatric symptoms in pain etiology and maintenance, the presence of psychiatric symptoms likely complicates chronic pain treatment. Anxiety, depressive mood, oppositional behaviors, and inattention/distractibility have the strong potential to interfere with youths' willingness and ability to fully engage in interventions within a rehabilitative model, such as frequent physical therapy exercises. The impact of psychiatric symptoms on treatment outcomes within ambulatory clinic settings is not known. Further examination of the differential prevalence of psychiatric symptoms among various pediatric chronic pain conditions, along with concurrent study of the relation between psychiatric symptoms on treatment success is critical for developing optimal treatment plans and effectively 
allocating services within interdisciplinary pediatric pain treatment settings.

The aims of this study were to

a) Compare the prevalence of psychiatric symptoms among youth with chronic neuropathic pain versus patients with non-neuropathic pain,

b) To compare the degree of psychiatric symptoms for groups of youth with chronic neuropathic pain and nonneuropathic pain to normative data and

c) To examine associations among psychiatric symptoms and clinically meaningful decreases in pain intensity over the course of treatment. We hypothesized that symptoms of generalized anxiety disorder and depression would be more prevalent among children and adolescents with neuropathic pain and mean symptoms for this group would be significantly greater than symptoms in normative samples. We also expected that higher symptom levels of generalized anxiety and depression would be associated with significantly less reduction in pain scores at the conclusion of treatment.

\section{Methods}

\section{Patients}

Participants in this study were all children between 8 and 18 years of age seen at a multidisciplinary pediatric chronic pain clinic associated with the Children's Hospital of Chicago Medical Center between January 2010 and June 2014. To be included, a participant's parent or guardian had to have filled out a Children or Adolescent Symptom Inventory-Parent (C/ASI-P) [13,14] form at the initial visit and have had at least one return visit to the clinic.

\section{Procedure}

The treatment team at the multidisciplinary pain clinic consisted of pain physicians, advanced practice nurses, and psychologists. Depending on the specific patient evaluation, the treatment plan consisted of non-opioid medication management, nerve blocks, physical therapy, and psychological intervention with a cognitive-behavioral orientation. Nerve blocks were not routinely performed for CRPS except to facilitate physical therapy in severe cases, but were more often performed for headaches, abdominal/chest pain, and non-CRPS limb pain. Psychological evaluation may have prompted ongoing psychological therapy with either a pain clinic psychologist or, more commonly, referral to a psychologist in the patient's community. Treatment goals largely included reduction in pain scores, reduction in disability, improvement in school attendance, increased participation in activities, and improvement in sleep.

\section{Measures}

A chart review was conducted to gather information on the child's age, sex, and Medicaid status as a proxy for socioeconomic status. Patients with a diagnosis of neuropathic pain (including
CRPS and other neuropathies) were included in the neuropathic pain group and other patients were designated as the comparison group. An experienced physician with expertise in pediatric chronic pain (R.S.) categorized the patients based on the patient's presenting diagnosis prior to performing analyses. This categorization was repeated for a random sample of 24 (21\%) of the patients by a second pediatric pain physician (D.K.) ( $\kappa=0.75$, $\mathrm{SE}=0.13$ )

An 11-point numeric rating scale (NRS) from "no pain" (0) to "worst possible pain" (10) was used to quantify pain intensity at each clinic visit [15]. NRS ratings at the first visit were used to measure initial pain intensity. Because the duration of treatment and number of visits varied, the NRS pain intensity rating obtained at the last visit during the designated review period was used to assess outcome. Duration of treatment was calculated as the number of weeks between the initial and final clinic visits.

The 97-item Child Symptom Inventory (CSI-P) (ages 6-11) or the 120-item Adolescent Symptom Inventory (ASI-P) (ages $12-18),[13,14,16]$ was completed by a parent at the time of the initial clinic visit. These are widely used, well-formed measures of child and adolescent behavioral and emotional problems. Parent-reports were used because no well-standardized selfreport measures were available for the younger children in the sample. Items were derived from DSM-IV diagnostic criteria. The parent rated the extent to which each symptom was present in their child on a 4-point rating scale from "never" (0) to "very often" (3). The sum of the scores for each is calculated to generate a total symptom count, which is converted to a standardized t-score. Results for the attention deficit/hyperactivity disorder (ADHD)-combined (inattentive and hyperactive/impulsive) type, generalized anxiety disorder (GAD), separation anxiety disorder (SAD), and major depressive disorder (MDD) scores were used in the current analysis. Overall reliability of symptom scales is acceptable to high for ADHD ( $\alpha=0.94$; test-retest $=0.79$ ), MDD ( $\alpha=0.74$; test-retest $=0.68), \operatorname{SAD}(\alpha=0.85$; test-retest $=0.57)$, and GAD scales ( $\alpha=0.74$; test-retest $=0.53$ ). Spanish-language versions of the CSI-P and ASI-P are available and were used by parents who required an interpreter for the visitor who requested materials in Spanish.

Preliminary analyses were conducted to determine whether the neuropathic and non-neuropathic groups differed on demographic variables. Diagnostic group differences in gender and Medicaid status were examined using chi-square analyses and differences in age were examined using a t-test. Subsequently, t-tests were conducted to determine whether there were pain diagnosis group differences on initial and final pain intensity scores and initial measures (t-scores) of child ADHD, conduct, anxiety, and depression measures. In addition, the neuropathic and non-neuropathic pain groups were both compared to normative data along these same measures. Effect sizes were calculated for significant differences and reported as Cohen's $\mathrm{d}$ for tests of mean differences. 
To allow for the determination of the independent contribution of each predictor variable with final pain intensity scores, a hierarchical regression analysis was then used to determine the independent association of each predictor with final pain intensity scores. The first step of this analysis included the variables of diagnostic group (neuropathic versus non-neuropathic pain), initial pain intensity level, total duration of treatment, and demographic variables and psychological symptom scales that were significantly different between diagnostic groups at the time of the initial visit (based on t-test results). The second step included the interaction terms for diagnostic group $\mathrm{x}$ significant psychological factors.

\section{Results}

\section{Population demographics}

Out of a total of 177 patients who were seen in the NAME REDACTED Chronic Pain Clinic over a 4-year period, the parents of 104 patients had completed a C/ASI and were included in the study. Of these, 48 had a diagnosis of neuropathic pain (neuropathic group) and 56 had a diagnosis for a pain condition that was not considered to be neuropathic in nature (comparison group). Patients in the neuropathic pain group primarily had a diagnosis of CRPS (77\%), but other patients had a variety of nonmalignant neuropathic pain (Table 1). Patients in the comparison group had a variety of diagnoses, but most had a primary diagnosis of musculoskeletal pain (48\%) and headache (23\%), or abdominal pain (18\%). The neuropathic and comparison groups were similar demographically in age, socioeconomic status, sex, and treatment duration (Table 2).

Table 1: Diagnoses by groups.

\begin{tabular}{|l|c|c|}
\hline \multirow{4}{*}{ Neuropathic Group } & Diagnosis & N(\%) \\
\cline { 2 - 3 } & CRPS & $31(76)$ \\
\cline { 2 - 3 } & Neuropathic - other & $8(20)$ \\
\hline \multirow{4}{*}{ Comparison Group } & Radiculopathy & $2(4)$ \\
\cline { 2 - 3 } & Musculoskeletal & $25(49)$ \\
\cline { 2 - 3 } & Fibromyalgia & $6(11)$ \\
\cline { 2 - 3 } & Abdominal/Pelvic & $9(18)$ \\
\cline { 2 - 3 } & Headache & $11(22)$ \\
\hline
\end{tabular}

Table 2: Study population demographics.

\begin{tabular}{|c|c|c|c|c|c|c|}
\hline & & $N(\%)$ & Average $(95 \% \mathrm{CI})$ & t-Statistic (df) & $x^{2}$ & $\mathbf{P}$ \\
\hline \multirow{2}{*}{ Female } & Neuropathic & $34(71)$ & & & 0.23 & 0.67 \\
\hline & Comparison & $42(75)$ & & & & \\
\hline \multirow{2}{*}{ Medicaid } & Neuropathic & $12(25)$ & & & 0.37 & 0.66 \\
\hline & Comparison & $17(30)$ & & & & \\
\hline \multirow{2}{*}{ Age (yr) } & Neuropathic & & $12.9(0.39)$ & $1.80(102)$ & & 0.53 \\
\hline & Comparison & & $13.9(0.36)$ & & & \\
\hline \multirow{2}{*}{$\begin{array}{c}\text { Treatment } \\
\text { Duration (mo) }\end{array}$} & Neuropathic & & $11.1(1.9)$ & $1.07(102)$ & & 0.48 \\
\hline & Comparison & & $12.5(2.0)$ & & & \\
\hline
\end{tabular}

\section{Comparison of psychiatric symptoms with normative} data

Table 3: Effect sizes compared to normative data as described by the severity index in the C/ASI.

\begin{tabular}{|c|c|c|}
\hline $\begin{array}{c}\text { Neuropathic Pain } \\
\text { Group }\end{array}$ & Effect Size, $^{\mathbf{a}}$ & P \\
\hline GAD & 0.62 & 0.003 \\
\hline SAD & 0.46 & 0.03 \\
\hline MDD & 1.22 & 0.0001 \\
\hline ADHD & 0.28 & 0.17 \\
\hline Comparison Group & & 0.0001 \\
\hline GAD & 1.19 & 0.04 \\
\hline SAD & 0.39 & 0.0001 \\
\hline MDD & 1.4 & 0.004 \\
\hline ADHD & 0.55 & \\
\hline
\end{tabular}

${ }^{a}$ Descriptors for magnitudes of Cohen's $\mathrm{d}$ : $\mathrm{d} \sim 0.2=$ small, $\mathrm{d} \sim$
$0.5=$ moderate, $\mathrm{d} \sim 0.8=$ large.

To estimate the level of elevation of the C/ASI scores in the sample, we compared these to normative data. The neuropathic pain and non-neuropathic pain groups were analyzed separately for each C/ASI scale. Both groups experienced significantly higher levels of psychological distress in terms of GAD, MDD, and SAD in comparison to normative data. Moreover, the comparison group also experienced higher levels of symptoms of ADHD (Table 3). Effect sizes were moderate to high for the neuropathic group for measures of GAD, SAD, and MDD, and for the comparison group on GAD, MDD, and ADHD.

\section{Diagnostic group differences on pain and psychiatric symptoms}

At the initial visit, the diagnostic groups had similar pain intensity scores on the NRS scale (Table 4). Diagnostic group differences were observed for the measures of ADHD and GAD, with the non-neuropathic pain group scoring significantly higher on both of these indices on initial presentation to the chronic 
pain clinic. For both GAD and ADHD, effect sizes were moderate in magnitude. Diagnostic groups did not differ on symptom levels of separation anxiety, depression, ODD, and conduct disorder. Although the diagnostic groups did not differ on initial pain intensity scores, there was a significant diagnostic group difference

Table 4: Psychiatric symptom levels by pain type. on final pain intensity scores: patients with neuropathic pain had lower final pain intensity scores than their peers with other types of chronic pain. The length of treatment at the multidisciplinary pain clinic was not significantly different between the groups.

\begin{tabular}{|c|c|c|c|}
\hline & \multicolumn{2}{|l|}{ Diagnostic Group } & \multirow{3}{*}{ t-Score } \\
\hline & Neuropathic Pain & Comparison & \\
\hline & $(\mathrm{N}=62)$ & $(N=52)$ & \\
\hline Initial Pain Score & $6.6(2.0)$ & $6.5(2.1)$ & -0.19 \\
\hline Final Pain Score & $4.0(2.7)$ & $5.3(3.0)$ & $2.1^{*}$ \\
\hline GAD & $55.8(8.6)$ & $61.9(10)$ & $3.3^{* *}$ \\
\hline SAD & $54.3(8.9)$ & $53.4(7.1)$ & -0.62 \\
\hline MDD & $63.1(11)$ & $64.9(11)$ & 0.8 \\
\hline ADHD & $52.3(5.3)$ & $55.3(9.0)$ & $2.1^{*}$ \\
\hline ODD & $52.4(6.3)$ & $54.2(7.0)$ & 1.4 \\
\hline $\mathrm{CD}$ & $52.1(5.5)$ & $51.9(5.8)$ & -0.24 \\
\hline Age (yr) & $13.0(2.7)$ & $13.9(2.7)$ & 1.8 \\
\hline Treatment duration (mo) & $10.5(10.9)$ & $13.4(11.9)$ & 1.1 \\
\hline
\end{tabular}

Average (standard deviation); ${ }^{*} \mathrm{P}<0.05 ;{ }^{*} \mathrm{P}<0.001$.

\section{Correlation of psychiatric symptoms with final pain score}

Higher final pain scores are significantly, but weakly, correlated with higher initial pain and generalized anxiety (Table 5). Other psychiatric symptoms were not correlated with final pain score.

Table 5: Correlation of psychiatric symptoms with final pain scores.

\begin{tabular}{|c|c|c|}
\hline & Correlation Coefficient, $\mathbf{r}$ & $\mathbf{P}$ \\
\hline Initial Pain Score & 0.37 & 0.018 \\
\hline GAD & 0.2 & 0.048 \\
\hline SAD & -0.11 & 0.51 \\
\hline MDD & 0.13 & 0.35 \\
\hline ADHD & 0.1 & 0.52 \\
\hline ODD & -0.005 & 0.97 \\
\hline CD & -0.059 & 0.79 \\
\hline $\begin{array}{c}\text { Treatment duration } \\
\text { (mo) }\end{array}$ & 0.12 & 0.36 \\
\hline
\end{tabular}

\section{Predictors of final pain score}

The results of the hierarchical regression analysis indicated that:

a. Higher initial pain scores were associated with higher final pain scores;

b. Neither diagnostic group, duration of treatment, or initial ADHD or GAD scores were significant independent predictors of the final pain intensity score. c. The interactions of diagnostic group with both GAD and ADHD scores were not significant.

\section{Discussion}

In this clinic-based sample of pediatric chronic pain patients, psychiatric symptoms, specifically GAD and ADHD, were less prevalent in patients with neuropathic pain than other types of pain presenting to a multi-disciplinary pain clinic. These results differ from prior studies of CRPS and neuropathic pain which supported the concept that psychological problems may play a particularly important role in the occurrence of CRPS. The methodology for this study differs from prior studies of the psychological characteristics of children and adolescents with CRPS in two important ways:

a. A larger sample size than most, which rely on case studies [10], or very small samples [11],

b. Other studies made comparisons to standardization samples without including control or comparison groups [1116]. The findings of the present study highlight the importance of considering the association of neuropathic pain and psychiatric symptoms in the context of other pain conditions and their association with different types of chronic pain.

A second compelling finding is that although no predictors of improved pain scores could be found, anxiety, but no other psychiatric symptoms, are correlated with higher final pain scores. These findings strengthen the notion that compared with normative samples; children with chronic pain have a greater incidence of comorbid psychiatric symptoms including GAD, 
SAD, ADHD, and MDD and GAD than their pain-free peers. The findings, however, suggest that psychological problems among patients with neuropathic pain is not elevated, and is sometimes lower, than for children with other forms of non-neuropathic pain. Additionally, GAD seems to have a more significant interplay with chronic pain as it may play a larger role for children with nonneuropathic pain than other symptoms and it is correlated with final pain scores. Furthermore, neuropathic pain patients may have a lower burden of psychological comorbidity than other types of pediatric chronic pain. Limitations of this study are the retrospective nature of the analysis and reliance on subjective pain scores as the sole condition of treatment response is another limitation.

\section{Conclusions}

In this cohort of children and adolescents with chronic pain we continue to see higher levels of psychological symptoms GAD, SAD, MDD, and ADHD than in a normative population. Additionally, there appear to be differences in these symptoms depending on the nature of the chronic pain (neuropathic or nonneuropathic). Children with non-neuropathic type pain exhibit higher levels of GAD and ADHD. Children with non-neuropathic type pain have higher pain scores at the end of treatment. Future research should continue to investigate how anxiety and other psychiatric symptoms may interplay with pain experience, trajectory, functional status, the ability to engage in long-term treatment paradigms and success of treatment.

\section{Declarations}

Ethics approval and consent to participate: The Institutional Review Board (IRB) of the Children's Hospital of Chicago Medical Center approved this retrospective cohort study. Because the study design included retrospective chart review, informed consent was not obtained, and the IRB granted a HIPAA waiver to collect and analyze the data.

\section{Consent for publication}

Not applicable.

\section{Availability of Data and Material}

The datasets generated and/or analyzed during the current study are not publicly available due to the retrospective nature of the study since consent was waived and the IRB did not approve public availability of patient datasets, but are available from the corresponding author on reasonable request.

\section{Competing Interests}

The authors declare that they have no competing interests.

\section{Funding}

The study was funded solely through departmental sources.

\section{Authors' contributions}

DK performed data analysis, interpretation of results and was a major contributor in writing and editing the manuscript. AH collected data and performed analysis and interpretation of data and results and was a contributor in writing the manuscript. RS conceptualized the study design, interpreted results and was a contributor to the editing of the manuscript. JL conceptualized the study design, analyzed data, interpreted results, and was a major contributor to the writing and editing of the manuscript. $\mathrm{BE}$ interpreted results and contributed to the editing of the manuscript. SS interpreted results and was a contributor to editing of the manuscript. All authors read and approved the final manuscript.

\section{Acknowledgements}

None.

\section{References}

1. Main CJ (2013) The importance of psychosocial influences on chronic pain. Pain management 3(6): 455-466.

2. Mangerud WL, Bjerkeset O, Lydersen S, Indredavik MS (2013) Chronic pain and pain-related disability across psychiatric disorders in a clinical adolescent sample. BMC psychiatry 13: 272.

3. Zernikow B, Wager J, Hechler T, Hasan C, Rohr U, et al. (2012) Characteristics of highly impaired children with severe chronic pain: A 5-year retrospective study on 2249 pediatric pain patients. BMC pediatrics 12: 54 .

4. Kashikar Zuck S, Goldschneider KR, Powers SW, Vaught MH, Hershey AD (2001) Depression and functional disability in chronic pediatric pain. Clin J Pain 17(4): 341-349.

5. Kashikar Zuck S, Parkins IS, Graham TB, Lynch AM, Passo M, et al. (2008) Anxiety, mood, and behavioral disorders among pediatric patients with juvenile fibromyalgia syndrome. Clin J Pain 24(7): 620626.

6. Knook LE, Konijnenberg A, van der Hoeven J, Kimpen JL, Buitelaar J, et al. (2011) Psychiatric disorders in children and adolescents presenting with unexplained chronic pain: What is the prevalence and clinical relevancy? Eur Child Adolesc Psychiatry 20(1): 39-48.

7. Eccleston C, Crombez G, Scotford A, Clinch J, Connell H (2004) Adolescent chronic pain: Patterns and predictors of emotional distress in adolescents with chronic pain and their parents. Pain 108(3): 221229.

8. Campo JV, Bridge J, Ehmann M, Altman S, Lucas A, et al. (2004) Recurrent abdominal pain, anxiety, and depression in primary care. Pediatrics 113(4): 817-824.

9. Pearson RD, Bailey J (2011) Complex regional pain syndrome in an 8-year-old female with emotional stress during deployment of a family member. Mil Med 176(8): 876-878.

10. Sherry DD, Weisman R (1988) Psychologic aspects of childhood reflex neurovascular dystrophy. Pediatrics 81: 572-578.

11. Cruz N, Reilly OJ, Slomine BS, Salorio CF (2011) Emotional and neuropsychological profiles of children with complex regional pain syndrome type-I in an inpatient rehabilitation setting. Clin J Pain 27(1): 27-34.

12. Logan DE, Williams SE, Carullo VP, Claar RL, Bruehl S, et al. (2013) Children and adolescents with complex regional pain syndrome: More 
psychologically distressed than other children in pain? Pain Res Manag 18(2): 87-93.

13. Gadow KD, Sprafkin J (1997) Early childhood inventory 4 norms manual. Stonybrook, Checkmate Plus, NY, USA.

14. Gadow KD, Sprafkin J (2000) Early childhood inventory 4 screening manual. Stonybrook, Checkmate Plus, NY, USA.
15. Bijur PE, Latimer CT, Gallagher EJ (2003) Validation of a verbally administered numerical rating scale of acute pain for use in the emergency department. Acad Emerg Med 10(4): 390-392.

16. Sprafkin J, Gadow KD, Salisbury H, Schneider J, Loney J (2002) Further evidence of reliability and validity of the child symptom inventory-4: Parent checklist in clinically referred boys. J Clin Child Adolesc Psychol 31(4): 513-524

This work is licensed under Creative Commons Attribution 4.0 License

DOI: $10.19080 / J A I C M .2018 .05 .555669$ 